\title{
Polarimetric survey of asteroids with the Asiago telescope ${ }^{\star}$
}

\author{
S. Fornasier ${ }^{1}$, I. N. Belskaya ${ }^{2}$, Yu. G. Shkuratov ${ }^{2}$, C. Pernechele ${ }^{3}$, C. Barbieri ${ }^{1}$, E. Giro ${ }^{4}$, and H. Navasardyan ${ }^{4}$ \\ 1 Dipartimento di Astronomia, Università di Padova, Italy \\ e-mail: fornasier@pd.astro.it \\ 2 Astronomical Institute of Kharkiv National University, Ukraine \\ 3 INAF - Osservatorio Astronomico di Cagliari, Italy \\ 4 INAF - Osservatorio Astronomico di Padova, Italy
}

Received 10 January 2006 / Accepted 24 March 2006

ABSTRACT

\begin{abstract}
Aims. We present the first results of an asteroid photo-polarimetry program started at Asiago-Cima Ekar Observatory. The aim of our survey is to estimate diversity in polarimetric properties of asteroids belonging to different taxonomic and dynamical classes. Methods. The data were obtained with the polarization analyser placed inside the Faint Object Spectrographic Camera (AFOSC) of the $1.8 \mathrm{~m}$ telescope. This instrument allows simultaneous measurements of the two first Stokes parameters without any $\lambda / 2$ retarding plate. Results. Our survey began in 2002, and up to now we have obtained data on a sample of 36 asteroids; most of them are being investigated with the polarimetric technique for the first time. Combining our data with those already available in literature, we present an estimate of the inversion angle for 7 asteroids in this paper. Furthermore, we present the polarimetric measurements of the rare asteroid classes belonging to the A and D types and a detailed VRI observations at extremely small phase angles of the low albedo asteroid 1021 Flammario
\end{abstract}

Key words. minor planets, asteroids - techniques: polarimetric

\section{Introduction}

It is well known that asteroids are characterized by various polarimetric properties which are similar within the same composition type (e.g. Muinonen et al. 2002). The asteroid belt consists indeed of bodies of different compositions, varying from primitive to strongly evolved. Primitive asteroids, such as the C-type, present a higher value of the polarization minimum and a smaller inversion angle, as compared for instance to S- or Etype members.

The polarization degree is defined as $P_{r}=\left(I_{\perp}-I_{\|}\right) /\left(I_{\perp}+I_{\|}\right)$, where $I_{\perp}$ and $I_{\|}$are respectively the intensity of the light scattered from the surface perpendicular to the polarization plane and from the surface parallel to the scattering plane. The polarization curves formed by plotting the polarization versus the phase angle prove to be an important source of information about the microstructure and composition of the asteroid surfaces.

Particularly interesting is the so-called negative branch of the polarization degree, in a range of phase angles between $0^{\circ}$ and about $18-20^{\circ}$, since it is found by laboratory measurements to be extremely dependent on the microstructure and composition of the surface. This negative branch has been studied for a long time by several authors and is interpreted with two main physical mechanisms, of which we report some recent investigations.

The first is related to the single particle scattering. It was shown experimentally (Volten et al. 2001; Shkuratov et al. 2004) and theoretically (Shkuratov et al. 2002; Zubko et al. 2005) that irregular particles (including fractal-like aggregates) reveal the negative polarization branch (with the depth of $1-10 \%$ ) in a rather wide range of particle sizes and optical constants. This

^ Based on observations carried out at the Asiago Astrophysical Observatory, Italy. polarization can survive, despite interparticle multiple scattering, even in the case of bright particulate surfaces. The reason of the negative polarization at single particle scattering has not yet been established.

The second mechanism of the negative polarization is associated with the coherent backscattering effect (e.g. Muinonen 2004). This effect is due to the constructive interference of timereversal trajectories of light multiply scattered in a particulate surface at small phase angles. This interference can contribute to the brightness opposition spike of asteroids, as well as to their negative polarization branch.

We note that the latter mechanism is more appropriate for bright objects, whereas the first one can provide the negative polarization in the total albedo range. The negative polarization strongly depends on surface albedo. Owing to that fact, polarimetry may give independent determinations of asteroid albedos, based on empirical relations that have been well-verified in laboratory experiments. In fact, empirical relations (Zellner \& Gradie 1976; Lupishko \& Mohamed 1996; Cellino et al. 1999) allow for the determination of the asteroid albedo, thanks to the knowledge of the minimum value $\left(P_{\min }\right)$ of polarization or/and of the slope parameter $h$ (i.e., the slope measured at the inversion angle $\alpha_{0}$ ). Polarimetric albedos are in agreement with data derived by other techniques, including direct measurements such as occultations and space missions (e.g. Lupishko \& Mohamed 1996).

Unfortunately, polarimetric observations are not easy, as polarimetric devices are offered by few telescopes. Moreover, an asteroid must be followed at different phase angles to investigate the polarization curve in detail, and this coverage becomes difficult, considering the limited visibility of a moving target and/or weather problems in the allocated nights. As a 

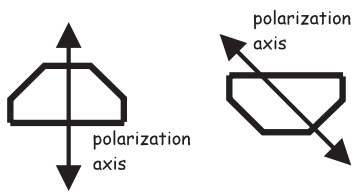

a)
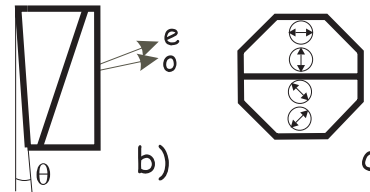

b)

c)

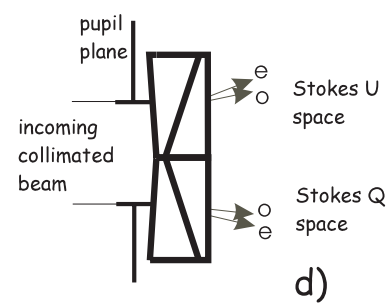

Fig. 1. Views of the double Wollaston prism: a) the two separated Wollaston half-prisms; b) an edge view of one of the two Wollastons to show the wedge shape of the prism and the exit direction of ordinary and extraordinary rays; c) the prism as seen face-on, with arrows indicating the transmitted polarization components; d) the two Wollastons glued together and mounted on the re-imaged pupil.

consequence, although in recent years many theoretical studies and laboratory models of asteroidal polarization properties have appeared, the data set on asteroid polarimetry has not been enlarged proportionally.

To increase this important database, we began a survey in 2002 using a $1.8 \mathrm{~m}$ telescope at the Asiago Astrophysical Observatory, that was recently equipped with a polarimeter device. In this paper, we present a description of the instrumentation together with our first results.

\section{The polarimeter of the Asiago Observatory}

The Asiago $1.82 \mathrm{~m}$ telescope is equipped with the Asiago Faint Object Spectrographic Camera (AFOSC) and a 1024×1024 pixel CCD, providing a total field of view of $8.14 \times 8.14$ arcmin. The pixel size is $24 \mu \mathrm{m}$ and the pixel scale is $0.473 \mathrm{arcsec} / \mathrm{px}$. Since 2001, an innovative polarimeter has been mounted on the AFOSC instrument, which allows us to simultaneously obtain images or spectra of the astronomical source, corresponding to the information of the polarization at $0^{\circ}, 45^{\circ}, 90^{\circ}$, and $135^{\circ}$, respectively. To obtain this, two Wollaston quartz prisms have been used, as is shown in Fig. 1.

The Wollaston prism is made of two pieces glued together along a plane surface perpendicular to the crystal axis of one of them and at $45^{\circ}$ to the axis of the other one (Figs. 1a, c). The front surface of each piece is cut to obtain a wedge, which deviates the rays above and below the optical axis (Fig. 1b). The double prism, mounted close to the re-imaged pupil, splits the incoming collimated beam into four beams polarized at $0,90,135$, and $45^{\circ}$ respectively (Figs. $1 \mathrm{c}, \mathrm{d}$ ). These four beams are sufficient to determine the first three elements of the Stokes vector, i.e., the intensity I and the two linear polarization parameters $Q$ and $U$.

Figure 2 illustrates how the polarimeter works on the collimated beam. The input field has been masked at the telescope

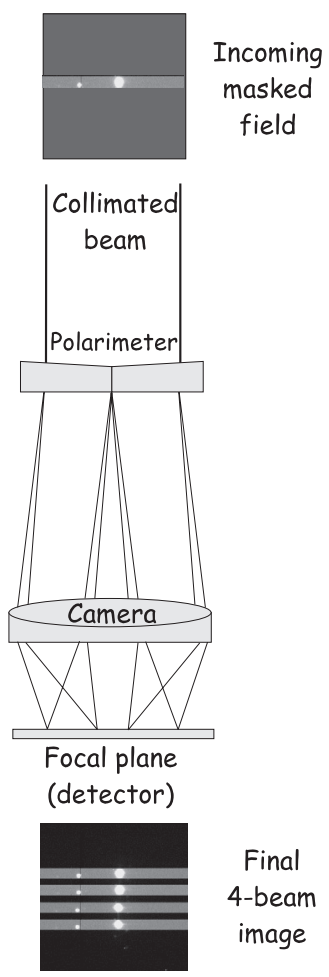

Fig. 2. A schematic optical layout showing how the double prism works, the collimator is not drawn. The light coming in from the polarimetric mask on the focal plane is split in four different beams by the polarization analyser. Finally the camera refocuses the beams on the detector.

focal plane by a field selective mask customized for imaging or spectropolarimetry. This is necessary to avoid the overlap of the final images on the CCD detector. The four output beams emerge collimated from the double Wollaston prism and are then focused on the CCD detector by the focal camera of AFOSC. The final image is formed by four strips from which the $I, Q$, and $U$ parameters can be extracted. The Asiago polarimeter, can also work in the spectro-polarimetric mode, with the Wollaston prisms mounted on the filter wheel, followed by the appropriate grism.

From the analysis of several unpolarized and polarized standard stars, it is seen that the instrumental polarization is fairly constant and always below $0.4 \%$, while the systematic errors in the position angle are below $1.5^{\circ}$ (Desidera et al. 2004).

The greater part of our observations have been obtained in the Johnson $V$-filter centred at $5474 \AA$, with a bandpass of $899 \AA$.

\section{Data acquisition and reduction}

The very first results on asteroids' photo-polarimetry were obtained in October 2002; subsequently, we had several observing runs allocated in service mode (see Tables 1 and 2).

The observational procedure includes the acquisition of several flat field images in two different sets corresponding to the adapter position of $0^{\circ}$ and $90^{\circ}$, to average the polarization induced by the reflections on the dome screen of the flat field lamp. Subtracting the bias, this average produces a master flat. Moreover, polarized and unpolarized standard stars are repeatedly acquired during each night to calibrate the instrumental polarization and the zero point of the position angles. The asteroids and standard star images are then corrected for bias and master 
Table 1. Results of the polarimetric observations in the $V$ filter. $\mathrm{Cl}$ is the spectral classification according to the Tholen taxonomy (Tholen 1989), while $\alpha$ and $\phi$ are, respectively, the phase and position angles.

\begin{tabular}{|c|c|c|c|c|c|c|c|c|c|}
\hline Asteroid & DATE & UT & $\phi\left({ }^{\circ}\right)$ & $\alpha\left(^{\circ}\right)$ & $P(\%)$ & $\theta\left(^{\circ}\right)$ & $P_{r}(\%)$ & $\theta_{r}\left({ }^{\circ}\right)$ & $\mathrm{Cl}$ \\
\hline 15 Eunomia & $2002-10-29$ & $20: 13$ & 80.4 & 21.4 & $0.09 \pm 0.02$ & $156.0 \pm 6.4$ & $+0.08 \pm 0.030$ & $165.6 \pm 6.4$ & $\mathrm{~S}$ \\
\hline 21 Lutetia & 2004-12-14 & $20: 58$ & 68.5 & 18.8 & $0.69 \pm 0.07$ & $86.1 \pm 2.9$ & $-0.56 \pm 0.07$ & $107.7 \pm 2.9$ & M \\
\hline 21 Lutetia & 2005-03-17 & $19: 18$ & 76.8 & 20.3 & $0.54 \pm 0.07$ & $60.6 \pm 3.7$ & $-0.46 \pm 0.07$ & $73.8 \pm 3.7$ & M \\
\hline 44 Nysa & $2002-10-30$ & $01: 15$ & 272.4 & 10.6 & $0.25 \pm 0.08$ & $100.5 \pm 9.0$ & $-0.24 \pm 0.08$ & $98.5 \pm 9.0$ & $\mathrm{E}$ \\
\hline 46 Hestia & 2002-10-29 & $23: 48$ & 268.0 & 5.1 & $1.38 \pm 0.08$ & $86.3 \pm 1.7$ & $-1.37 \pm 0.09$ & $88.3 \pm 1.7$ & $\mathrm{P}$ \\
\hline 71 Niobe & 2003-09-27 & $00: 19$ & 122.6 & 8.2 & $0.39 \pm 0.09$ & $113.3 \pm 6.6$ & $-0.37 \pm 0.09$ & $80.7 \pm 6.6$ & S \\
\hline 71 Niobe & 2003-12-14 & 20:05 & 65.1 & 18.3 & $0.12 \pm 0.03$ & $159.1 \pm 7.1$ & $+0.12 \pm 0.03$ & $3.9 \pm 7.1$ & $\mathrm{~S}$ \\
\hline 102 Miriam & $2003-12-15$ & 00:02 & 339.6 & 2.9 & $0.72 \pm 0.03$ & $160.7 \pm 1.3$ & $-0.72 \pm 0.03$ & $91.1 \pm 1.3$ & $\mathrm{C}$ \\
\hline 110 Lydia & 2003-11-18 & $23: 34$ & 65.4 & 6.3 & $0.82 \pm 0.04$ & $62.6 \pm 1.4$ & $-0.82 \pm 0.04$ & $87.3 \pm 1.4$ & M \\
\hline 110 Lydia & $2003-12-14$ & $21: 39$ & 69.9 & 15.6 & $0.56 \pm 0.04$ & $70.6 \pm 2.0$ & $-0.56 \pm 0.04$ & $90.7 \pm 2.0$ & M \\
\hline 140 Siwa & 2003-02-12 & $23: 32$ & 163.2 & 1.0 & $0.51 \pm 0.06$ & $148.1 \pm 3.3$ & $-0.44 \pm 0.07$ & $74.9 \pm 3.3$ & $\mathrm{P}$ \\
\hline 140 Siwa & $2005-10-28$ & $00: 50$ & 25.1 & 2.7 & $0.57 \pm 0.06$ & $35.8 \pm 3.0$ & $-0.53 \pm 0.06$ & $100.7 \pm 3.0$ & $\mathrm{P}$ \\
\hline 152 Atala & 2003-11-19 & $00: 12$ & 77.2 & 5.3 & $0.37 \pm 0.04$ & $70.4 \pm 3.9$ & $-0.37 \pm 0.04$ & $87.0 \pm 3.9$ & $\mathrm{D} / \mathrm{S}$ \\
\hline 152 Atala & 2003-12-14 & $22: 50$ & 73.4 & 13.7 & $0.14 \pm 0.05$ & $67.3 \pm 10.0$ & $-0.14 \pm 0.07$ & $83.9 \pm 10.0$ & $\mathrm{~S}$ \\
\hline 153 Hilda & $2003-12-15$ & 03:34 & 293.9 & 5.3 & $0.90 \pm 0.10$ & $108.3 \pm 3.2$ & $-0.88 \pm 0.10$ & $84.4 \pm 3.2$ & $X$ \\
\hline 165 Lorely & 2004-12-14 & $18: 35$ & 69.9 & 17.6 & $0.53 \pm 0.08$ & $109.8 \pm 4.3$ & $-0.09 \pm 0.08$ & $129.9 \pm 4.3$ & $\mathrm{C} / \mathrm{D}$ \\
\hline 171 Ophelia & 2003-11-19 & 01:01 & 57.2 & 3.04 & $0.63 \pm 0.03$ & $57.8 \pm 1.4$ & $-0.63 \pm 0.04$ & $90.6 \pm 1.4$ & $\mathrm{C}$ \\
\hline 208 Lacrimosa & 2002-10-29 & $23: 15$ & 101.0 & 0.9 & $0.31 \pm 0.09$ & $105.7 \pm 8.3$ & $-0.30 \pm 0.010$ & $94.7 \pm 8.3$ & $\mathrm{~S}$ \\
\hline 214 Aschera & 2004-12-14 & $17: 26$ & 67.2 & 21.7 & $0.46 \pm 0.05$ & $133.0 \pm 3.1$ & $+0.31 \pm 0.05$ & $155.8 \pm 3.1$ & E \\
\hline 225 Henrietta & 2004-12-15 & $03: 27$ & 298.0 & 12.8 & $1.30 \pm 0.14$ & $122.0 \pm 3.1$ & $-1.29 \pm 0.14$ & $94.0 \pm 3.1$ & $\mathrm{~F}$ \\
\hline 250 Bettina & 2003-11-19 & 02:09 & 106.7 & 4.8 & $0.78 \pm 0.06$ & $104.9 \pm 2.2$ & $-0.78 \pm 0.06$ & $88.2 \pm 2.2$ & M \\
\hline 365 Corduba & 2003-09-27 & 01:51 & 86.8 & 2.33 & $0.84 \pm 0.03$ & $88.1 \pm 1.1$ & $-0.84 \pm 0.03$ & $91.3 \pm 1.1$ & $\mathrm{C}$ \\
\hline 377 Campania & 2003-09-26 & $23: 29$ & 81.8 & 11.5 & $1.76 \pm 0.06$ & $76.1 \pm 1.0$ & $-1.72 \pm 0.06$ & $84.3 \pm 1.0$ & $\mathrm{C}$ \\
\hline 377 Campania & $2003-12-13$ & 18:03 & 67.30 & 22.5 & $0.56 \pm 0.05$ & $159.3 \pm 2.5$ & $+0.55 \pm 0.05$ & $1.9 \pm 2.5$ & $\mathrm{C}$ \\
\hline 381 Myrrha & 2003-01-30 & $23: 47$ & 177.0 & 0.5 & $0.44 \pm 0.04$ & $157.9 \pm 2.6$ & $-0.34 \pm 0.04$ & $70.9 \pm 2.6$ & $\mathrm{C}$ \\
\hline 386 Siegena & 2004-05-27 & 00:56 & 228.1 & 12.2 & $1.47 \pm 0.07$ & $46.8 \pm 1.4$ & $-1.46 \pm 0.07$ & $88.8 \pm 1.4$ & $\mathrm{C}$ \\
\hline 386 Siegena & 2004-07-10 & $01: 21$ & 141.4 & 11.1 & $1.53 \pm 0.05$ & $144.8 \pm 1.0$ & $-1.52 \pm 0.05$ & $93.4 \pm 1.0$ & $\mathrm{C}$ \\
\hline 409 Aspasia & $2002-10-29$ & $22: 45$ & 95.1 & 7.7 & $1.56 \pm 0.05$ & $95.2 \pm 1.0$ & $-1.56 \pm 0.05$ & $90.1 \pm 1.0$ & CX \\
\hline 409 Aspasia & 2003-12-15 & $05: 15$ & 296.3 & 14.4 & $1.04 \pm 0.07$ & $115.1 \pm 2.0$ & $-1.04 \pm 0.07$ & $88.8 \pm 2.0$ & CX \\
\hline 420 Bertholda & 2004-09-18 & $23: 14$ & 237.3 & 8.7 & $1.03 \pm 0.05$ & $58.5 \pm 1.4$ & $-1.03 \pm 0.05$ & $91.2 \pm 1.4$ & $\mathrm{P}$ \\
\hline 438 Zeuxo & 2004-09-19 & 01:29 & 256.6 & 15.2 & $0.83 \pm 0.05$ & $64.6 \pm 1.7$ & $-0.76 \pm 0.05$ & $78.1 \pm 1.7$ & $\mathrm{~F}$ \\
\hline 456 Abnoba & 2003-09-26 & $21: 18$ & 100.1 & 11.2 & $0.64 \pm 0.08$ & $95.1 \pm 3.6$ & $-0.63 \pm 0.08$ & \pm 3.6 & $\mathrm{~S}$ \\
\hline 456 Abnoba & 2004-12-14 & 23:06 & 70.0 & 10.1 & $0.66 \pm 0.08$ & $80.5 \pm 3.5$ & $-0.61 \pm 0.08$ & $100.5 \pm 3.5$ & $\mathrm{~S}$ \\
\hline 466 Tisiphone & 2003-09-26 & 22:09 & 111.1 & 7.9 & $1.19 \pm 0.10$ & $104.8 \pm 2.4$ & $-1.16 \pm 0.10$ & $83.7 \pm 2.4$ & $\mathrm{C}$ \\
\hline 466 Tisiphone & 2003-12-14 & $18: 54$ & 64.4 & 15.5 & $0.96 \pm 0.12$ & $59.5 \pm 3.6$ & $-0.95 \pm 0.12$ & $85.1 \pm 3.6$ & $\mathrm{C}$ \\
\hline 466 Tisiphone & 2004-12-14 & $22: 19$ & 91.1 & 11.6 & $1.46 \pm 0.08$ & $96.5 \pm 1.6$ & $-1.44 \pm 0.08$ & $95.4 \pm 1.6$ & $\mathrm{C}$ \\
\hline 466 Tisiphone & $2005-10-28$ & $02: 30$ & 282.1 & 17.6 & $0.77 \pm 0.09$ & $93.6 \pm 3.3$ & $-0.74 \pm 0.09$ & $81.5 \pm 3.3$ & $\mathrm{C}$ \\
\hline 762 Pulcova & 2003-11-18 & $22: 34$ & 77.6 & 14.1 & $0.79 \pm 0.08$ & $77.8 \pm 2.9$ & $-0.79 \pm 0.08$ & $90.1 \pm 2.9$ & $\mathrm{~F}$ \\
\hline 762 Pulcova & 2004-12-15 & $00: 26$ & 232.1 & 5.6 & $0.77 \pm 0.05$ & $66.3 \pm 1.9$ & $-0.68 \pm 0.05$ & $104.2 \pm 1.9$ & $\mathrm{~F}$ \\
\hline 785 Zwetana & 2003-11-19 & $03: 14$ & 273.1 & 4.23 & $0.81 \pm 0.04$ & $95.2 \pm 1.5$ & $-0.81 \pm 0.04$ & $92.1 \pm 1.5$ & M \\
\hline 785 Zwetana & 2005-03-18 & $01: 34$ & 266.5 & 18.7 & $0.98 \pm 0.05$ & $75.6 \pm 1.5$ & $-0.91 \pm 0.050$ & $79.2 \pm 1.5$ & M \\
\hline 863 Benkoela & $2003-05-25$ & 01:33 & 151.5 & 13.7 & $0.28 \pm 0.07$ & $158.2 \pm 7.0$ & $-0.27 \pm 0.07$ & $96.7 \pm 7.0$ & A \\
\hline 863 Benkoela & 2004-07-09 & $21: 45$ & 116.4 & 18.8 & $0.08 \pm 0.04$ & $54.7 \pm 14.0$ & $+0.04 \pm 0.04$ & $28.3 \pm 14.0$ & A \\
\hline 893 Leopoldina & 2004-07-09 & $23: 55$ & 181.0 & 5.4 & $1.06 \pm 0.06$ & $176.5 \pm 1.6$ & $-1.05 \pm 0.06$ & $85.5 \pm 1.6$ & $\mathrm{XF}$ \\
\hline 925 Alphonsina & 2002-10-29 & $18: 10$ & 78.6 & 18.5 & $0.07 \pm 0.04$ & $86.0 \pm 16.0$ & $-0.07 \pm 0.04$ & $98.2 \pm 16.0$ & $\mathrm{~S}$ \\
\hline 925 Alphonsina & 2003-12-15 & $01: 37$ & 173.0 & 7.9 & $0.76 \pm 0.04$ & $171.2 \pm 1.5$ & $-0.76 \pm 0.04$ & $88.2 \pm 1.5$ & $\mathrm{~S}$ \\
\hline 944 Hidalgo & 2004-12-14 & $19: 30$ & 80.5 & 26.8 & $2.52 \pm 0.04$ & $165.8 \pm 0.5$ & $+2.49 \pm 0.04$ & $175.4 \pm 0.5$ & $\mathrm{D}$ \\
\hline 1021 Flammario & 2004-12-15 & $02: 44$ & 288 & 16.4 & $0.37 \pm 0.13$ & $109.4 \pm 10.0$ & $-0.37 \pm 0.13$ & $91.4 \pm 10.0$ & $\mathrm{~F}$ \\
\hline 1021 Flammario & 2005-03-09 & $21: 21$ & 101.6 & 20.2 & $1.06 \pm 0.06$ & $4.4 \pm 1.6$ & $+1.02 \pm 0.06$ & $172.8 \pm 1.6$ & $\mathrm{~F}$ \\
\hline 1251 Hedera & 2004-12-15 & $05: 15$ & 290.3 & 17.0 & $0.21 \pm 0.12$ & $124.5 \pm 15.0$ & $-0.19 \pm 0.12$ & $104.2 \pm 15.0$ & E \\
\hline 1627 Ivar & 2005-03-14 & $23: 23$ & 135.4 & 8.7 & $0.47 \pm 0.08$ & $135.4 \pm 4.8$ & $-0.47 \pm 0.08$ & $90.0 \pm 4.8$ & $\mathrm{~S}$ \\
\hline 1627 Ivar & 2005-03-15 & $23: 35$ & 133.9 & 9.2 & $0.47 \pm 0.10$ & $116.0 \pm 6.0$ & $-0.38 \pm 0.10$ & $72.1 \pm 6.0$ & $\mathrm{~S}$ \\
\hline 1627 Ivar & 2005-03-17 & $20: 45$ & 131.5 & 10.3 & $0.32 \pm 0.05$ & $148.0 \pm 4.5$ & $-0.26 \pm 0.04$ & $106.5 \pm 4.5$ & $\mathrm{~S}$ \\
\hline 2797 Teucer & 2004-07-09 & $20: 28$ & 110.0 & 9.9 & $1.59 \pm 0.10$ & $126.3 \pm 1.8$ & $-1.34 \pm 0.10$ & $106.3 \pm 1.8$ & D \\
\hline 3200 Phaeton & 2004-12-14 & $21: 27$ & 92.7 & 23.0 & $0.54 \pm 0.10$ & $163.2 \pm 5.3$ & $0.43 \pm 0.10$ & $161.2 \pm 5.3$ & $\mathrm{~F}$ \\
\hline
\end{tabular}

flat, and the cosmic rays removed. The data reduction is performed by using dedicated software written in IDL.

The centre of the asteroid image in each of the four channels is evaluated by a 2 dimensional centroid algorithm. The flux for each channel is integrated over a radius corresponding to 3-4 times the average seeing, and the sky is subtracted using a $3-5$ pixel wide annulus around the asteroid. Naming the flux corresponding to the channels at $0,90,45$, and $135^{\circ}$ of polarization
$I_{0}, I_{90}, I_{45}$, and $I_{135}$, the Stokes parameters are then derived in the following manner:

$$
\begin{aligned}
& Q=\frac{I_{90}-I_{0}}{I_{90}+I_{0}} \\
& U=\frac{I_{135}-I_{45}}{I_{135}+I_{45}} .
\end{aligned}
$$


Table 2. Observations at small phase angles of asteroid 1021 Flammario in the $V, R$, and $I$ filters.

\begin{tabular}{lccccccc}
\hline \hline DATE & $\mathrm{UT}$ & $\phi\left({ }^{\circ}\right)$ & $\alpha\left(^{\circ}\right)$ & $P(\%)$ & $\theta\left(^{\circ}\right)$ & $P_{r}(\%)$ & $\theta_{r}\left({ }^{\circ}\right)$ \\
\hline$V$ filter & & & & & & & \\
\hline $2005-01-14$ & $23: 12$ & 284.5 & 0.6 & $0.12 \pm 0.04$ & $50.0 \pm 10.0$ & $+0.039 \pm 0.060$ & $35.5 \pm 10.0$ \\
$2005-01-15$ & $03: 08$ & 284.0 & 0.5 & $0.14 \pm 0.05$ & $53.5 \pm 6.0$ & $+0.027 \pm 0.05$ & $39.5 \pm 6.0$ \\
$2005-01-15$ & $21: 00$ & 272.2 & 0.1 & $0.10 \pm 0.05$ & $22.8 \pm 5.0$ & $+0.075 \pm 0.05$ & $20.6 \pm 5.0$ \\
$2005-01-16$ & $20: 39$ & 109.9 & 0.4 & $0.23 \pm 0.05$ & $38.2 \pm 5.0$ & $+0.178 \pm 0.05$ & $18.3 \pm 5.0$ \\
$2005-01-17$ & $02: 15$ & 109.1 & 0.6 & $0.10 \pm 0.05$ & $33.3 \pm 5.0$ & $+0.053 \pm 0.05$ & $14.2 \pm 5.0$ \\
$2005-01-17$ & $22: 30$ & 108.0 & 1.0 & $0.17 \pm 0.05$ & $63.4 \pm 5.0$ & $-0.003 \pm 0.05$ & $45.4 \pm 5.0$ \\
\hline$R$ filter & & & & & & & \\
\hline $2005-01-14$ & $23: 22$ & 284.5 & 0.6 & $0.129 \pm 0.06$ & $39.9 \pm 10.0$ & $+0.081 \pm 0.08$ & $25.4 \pm 10.0$ \\
$2005-01-15$ & $03: 21$ & 284.0 & 0.5 & $0.101 \pm 0.05$ & $36.2 \pm 7.0$ & $+0.072 \pm 0.05$ & $22.3 \pm 7.0$ \\
$2005-01-15$ & $21: 58$ & 272.2 & 0.1 & $0.258 \pm 0.05$ & $30.0 \pm 5.0$ & $+0.146 \pm 0.05$ & $27.8 \pm 5.0$ \\
$2005-01-16$ & $20: 52$ & 109.9 & 0.4 & $0.111 \pm 0.05$ & $157.6 \pm 5.0$ & $+0.010 \pm 0.05$ & $137.6 \pm 5.0$ \\
$2005-01-17$ & $02: 27$ & 109.1 & 0.6 & $0.114 \pm 0.06$ & $22.9 \pm 5.0$ & $+0.113 \pm 0.06$ & $3.8 \pm 5.0$ \\
$2005-01-17$ & $22: 37$ & 108.0 & 1.0 & $0.089 \pm 0.05$ & $65.7 \pm 5.0$ & $-0.008 \pm 0.05$ & $47.7 \pm 5.0$ \\
\hline$I$ filter & & \multicolumn{7}{c}{} & & & \\
\hline $2005-01-14$ & $23: 33$ & 284.5 & 0.6 & $0.137 \pm 0.05$ & $51.6 \pm 10.0$ & $+0.038 \pm 0.07$ & $37.1 \pm 10.0$ \\
$2005-01-15$ & $03: 33$ & 284.0 & 0.5 & $0.258 \pm 0.09$ & $39.4 \pm 5.0$ & $+0.163 \pm 0.11$ & $25.4 .0 \pm 5.0$ \\
$2005-01-16$ & $21: 01$ & 109.9 & 0.4 & $0.147 \pm 0.08$ & $26.5 \pm 5.0$ & $+0.143 \pm 0.09$ & $6.7 \pm 5.0$ \\
$2005-01-17$ & $22: 46$ & 108.0 & 1.0 & $0.118 \pm 0.06$ & $67.2 \pm 5.0$ & $-0.017 \pm 0.06$ & $49.2 \pm 5.0$ \\
\hline
\end{tabular}

The degree of polarization $P$ and the position angle $\theta$ of the polarization plane in the instrumental reference system are expressed via the parameters $Q$ and $U$ with the well-known formulae

$P=\sqrt{U^{2}+Q^{2}}$

$\theta=\frac{1}{2} \arctan \frac{U}{Q}$

The errors are evaluated in the following manner:

$\sigma_{P}=\frac{|U * \mathrm{~d} U+Q * \mathrm{~d} Q|}{P}$

and

$\sigma_{\theta}=\frac{28.65 * \sigma_{P}}{P}$

where $\mathrm{d} U$ and $\mathrm{d} Q$ are the errors on the Stokes parameters (Shakhovskoy \& Efimov 1972).

The position angle of the polarization plane relative to the plane perpendicular to the scattering plane, $\theta_{r}$, is expressed in terms of the position angle $\phi$ as

$\theta_{r}=\theta-\left(\phi \pm 90^{\circ}\right)$

where the sign inside the bracket is chosen to assure the condition $0^{\circ} \leq\left(\phi \pm 90^{\circ}\right) \leq 180^{\circ}$. The polarization quantity $P_{r}$ is derived from

$P_{r}=P * \cos \left(2 \theta_{r}\right)$

Each asteroid has been observed consecutively at least 3-5 times with an exposure time long enough to reach a high signal-tonoise ratio $(>200)$ in photon counts. The values reported in Tables 1 and 2 are the means of the results of these multiple exposures.

\section{Results}

We obtained data on 36 asteroids during 21 nights in 2002-2005 (Tables 1 and 2), with the aims of estimating diversity in polarimetric properties, and thus in surface characteristics. The choice of targets was made to cover asteroids of different composition and dynamical properties, which were not previously observed by the polarimetric technique.

Although most targets could be observed at a single phase angle, when comparing the data obtained with the available polarimetric measurements of asteroids of the same taxonomic type, we can draw interesting conclusions on the diversity of their surface properties.

Our approach is based on previous analyses of asteroidal polarization phase curves, which showed similar behaviour for asteroids of the same taxonomic class (e.g. Penttila et al. 2005). The available database on asteroid polarimetric observations (http://pdssbn.astro.umd.edu/sbnhtml/ asteroids), which contains data on about 100 asteroids of known taxonomic classes, has been used to supplement our observations and derive the polarimetric parameters. These parameters are given in Table 3, which contains asteroid type, IRAS albedo, $P_{\min }$, inversion angle $\alpha_{\text {inv }}$, number of points used to estimate polarimetric parameters, polarimetric slope, and polarimetric albedo defined using the slope-albedo or Pmin-albedo correlations adopted by Zellner \& Gradie (1976).

\subsection{S-type asteroids}

Seven S-type asteroids have been observed. Asteroids 15 Eunomia, 208 Lacrimosa, 456 Abnoba, and 925 Alphonsina have polarization values within the range typical for the S-type, while asteroids 71 Niobe, 152 Atala, and 1627 Ivar are characterized by substantially low polarization degrees (Fig. 3). Low polarization degrees imply high albedos of the surfaces of the above-mentioned asteroids. Indeed, the IRAS albedo of 71 Niobe is 0.3 , and its spectral properties are close to the E-type (Xe class according to Bus \& Binzel 2002). The albedo of 152 Atala is unknown, and there is a discrepancy in its classification: D (Tholen 1989) and S (Bus \& Binzel 2002). 
Table 3. Estimated polarimetric parameters for the asteroids which have been observed at different phase angles from this survey and literature data (IRAS albedos are taken from Tedesco et al. 2002).

\begin{tabular}{lccccccc}
\hline \hline Asteroid & TYPE & IRAS albedo & $\left|P_{\min }\right|$ & $\alpha_{\text {inv }}\left({ }^{\circ}\right)$ & $N$ & Slope (\%/deg) & Polarimetric albedo \\
\hline 15 Eunomia & S & 0.21 & - & 20.6 & 4 & 0.087 & 0.19 \\
21 Lutetia & M & 0.22 & - & 24.2 & 8 & 0.157 & 0.11 \\
71 Niobe & S, Xe & 0.31 & - & $<18$ & 3 & 0.061 & 0.27 \\
377 Campania & PD & 0.059 & 1.76 & 19.8 & 2 & 0.206 & 0.08 \\
409 Aspasia & CX & 0.061 & $>1.56$ & 19.9 & 4 & 0.191 & 0.09 \\
466 Tisiphone & $\mathrm{C}$ & 0.063 & 1.6 & - & 3 & - & 0.07 \\
863 Benkoela & $\mathrm{A}$ & 0.595 & 0.4 & 18.1 & 7 & 0.052 & 0.32 \\
925 Alphonsina & $\mathrm{S}$ & 0.28 & - & 19.6 & 2 & 0.065 & 0.26 \\
\hline
\end{tabular}

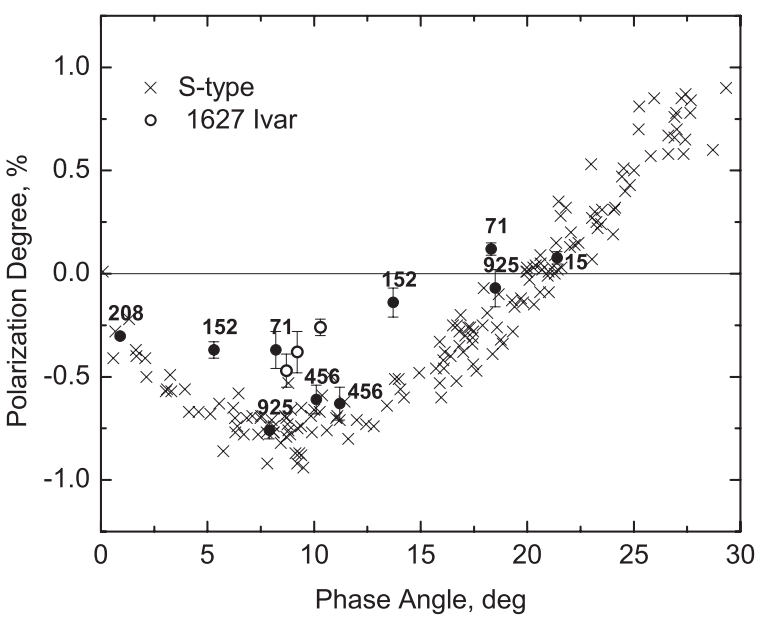

Fig. 3. Polarization phase curve for the S-type asteroids. Data marked by crosses are taken from Zellner \& Gradie (1976), Belskaya et al. (2003), Broglia \& Manara (1990, 1992), and Broglia et al. (1994).

Our polarimetric observations are in favor of a higher albedo than is typical for the $\mathrm{S}$ class.

Regarding the near-Earth asteroid 1627 Ivar, the polarization degree measured near the minimum of the negative polarization branch corresponds to an albedo of about 0.3, which is larger than that derived from the polarimetric slope (Kiselev et al. 1994) and thermal observations (Delbo et al. 2003). Ivar becomes the third S-type near-Earth asteroid for which negative polarization has been measured. Two other asteroids, 433 Eros and 1025 Ganymed, have larger negative polarization values typical of the S-type main belt asteroids.

\subsection{M-type asteroids}

Polarization degrees typical for the M-type asteroids have been measured for 110 Lydia and 250 Bettina (Fig. 4). Asteroid 785 Zwetana is characterized by a noticeable negative polarization at the phase angle of $18.7^{\circ}$, implying a large inversion angle. Its polarization is close to that of 21 Lutetia, an asteroid classified as M-type on the basis of its high IRAS albedo. However, considering the polarimetric properties (Belskaya \& Lagerkvist 1996), the lower radar albedo compared to the IRAS one (Magri et al. 1999), the $3 \mu \mathrm{m}$ absorption feature diagnostic of water of hydration (Rivkin et al. 2000), and a spectral behaviour in the near infrared region similar to that of the carbonaceous chondrites (Birlan et al. 2004; Barucci et al. 2005), 21 Lutetia probably does not belong to the $\mathrm{M}$ class. The taxonomic classification is also controversial for 785 Zwetana, due to its peculiar large inversion angle compared to that of the typical M-type asteroids. Further

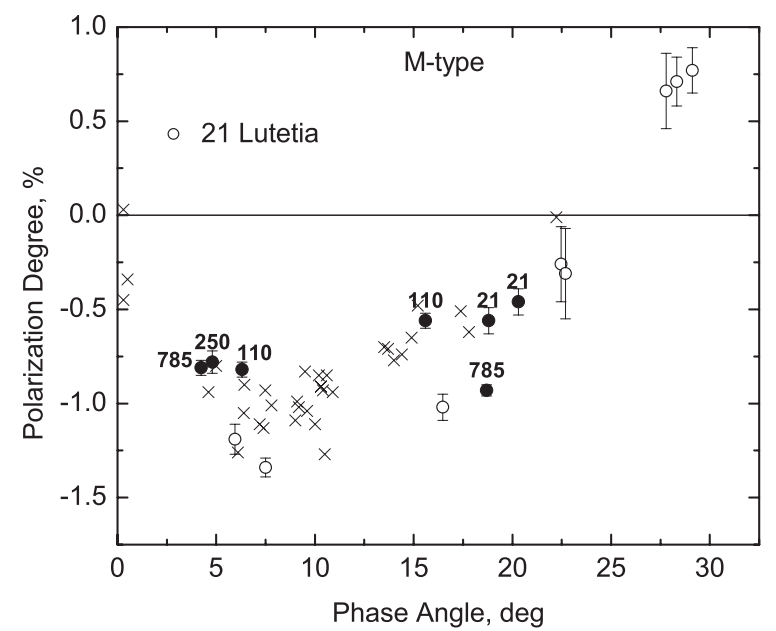

Fig. 4. Polarization phase curve for the M-type asteroids. Data marked by crosses and open circles are taken from Belskaya et al. (1985, 1987a, 1991), Broglia \& Manara (1992), Lupishko et al. (1994), and Zellner \& Gradie (1976).

observations covering different phase angle aspects are needed to fully understand the physical properties of this asteroid.

\subsection{E- and A-type asteroids}

We obtained polarimetric data for the E-type asteroids 214 Aschera and 1251 Hedera at a single phase angle (at $21.7^{\circ}$ and $17.0^{\circ}$, respectively), close to the inversion angle of polarization. The measured polarization values for both asteroids are typical for the E class (e.g. Zellner \& Gradie 1976; Fornasier et al. 2006) and seem to indicate that the inversion angle is in the region of $18-19^{\circ}$.

We have obtained the first polarimetric observations of an A-type asteroid. We observed 863 Benkoela as did Cellino et al. (2005), independently. The observations supplement each other (see Fig. 5) and allow us to determine the inversion angle and polarimetric slope. The observed polarization-phase behaviour of Benkoela is similar to that of the E-type asteroids. The estimated polarimetric albedo is $0.32 \pm 0.12$ lower than its IRAS albedo (see Table 3).

\subsection{Low albedo asteroids}

Asteroids of C- B-, F-, G-, and P-types have been previously observed. However, very few objects have been observed in each class, and thus it is difficult to distinguish between their polarimetric properties, with the notable exception of the F-type asteroids (see Fig. 6). Recently, it was shown that observed F-type 


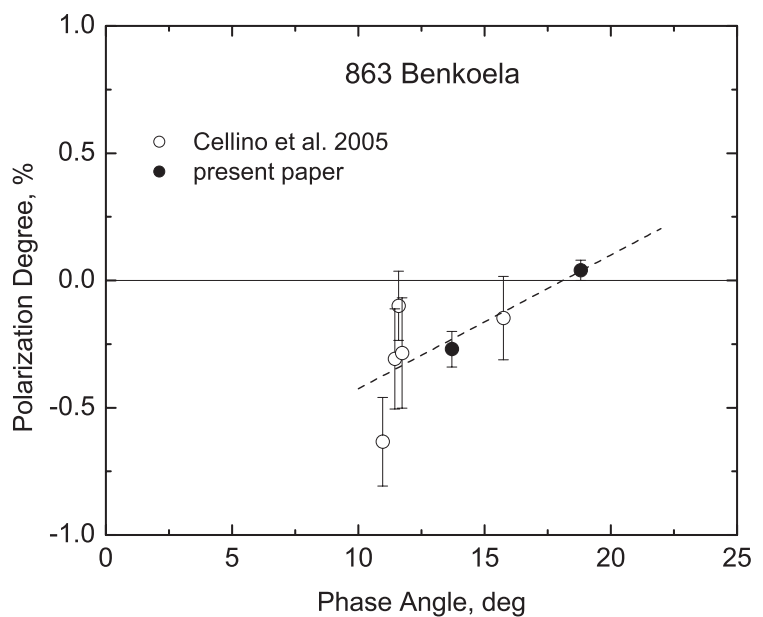

Fig. 5. Polarization phase curve for the A-type asteroid 863 Benkoela.

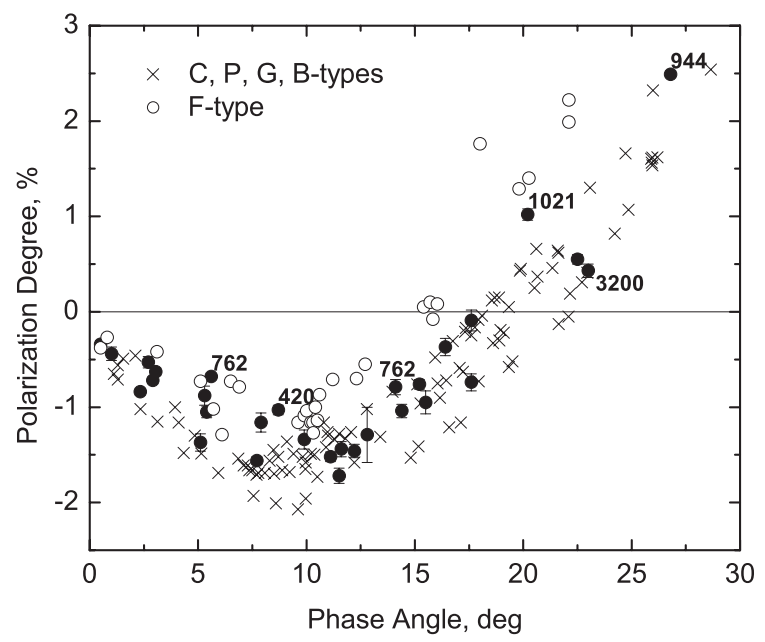

Fig. 6. Polarization phase curve for low albedo asteroids. Data marked by crosses and open circles are taken from Zellner \& Gradie (1976), Belskaya et al. (2003, 2005), Chernova et al. (1994), Lupishko et al. (1994), and Cellino et al. (2005).

asteroids are characterized by substantially smaller depths of the negative polarization branch, as compared to other low albedo asteroids. In addition, they have unusually small inversion angles (Belskaya et al. 2005). Therefore, we have included several F-asteroids in our sample to check whether the low inversion angles are inherent to all F-type asteroids. We find that 1021 Flammario has a smaller inversion angle than is typical for asteroids. At the same time, our observations of the nearEarth asteroid 3200 Phaethon, classified as F-type by Tholen (1985), definitely exclude a small inversion angle for it (see Fig. 6). Note, however, that there is an uncertainty in the classification of this asteroid. Judging from its relatively high IRAS albedo (0.098), Phaethon probably belongs to the B class (Bus \& Binzel 2002). Moreover, Licandro et al. (2005) found an absorption in the UV spectra of Phaethon inconsistent with the F class.

Our polarimetric observations of other F-type asteroids, 225 Henrietta, 438 Zeuxo, 762 Pulcova, and 893 Leopoldina, do not give definite estimates of their inversion angles. We can only conclude that the inversion angles of asteroids 438 Zeuxo and 762 Pulcova are larger than $16^{\circ}$.

Among the other low albedo asteroids observed in our survey, an interesting case is represented by the P-type asteroid 420 Bertholda, which shows negative polarization of $1.03 \%$ at

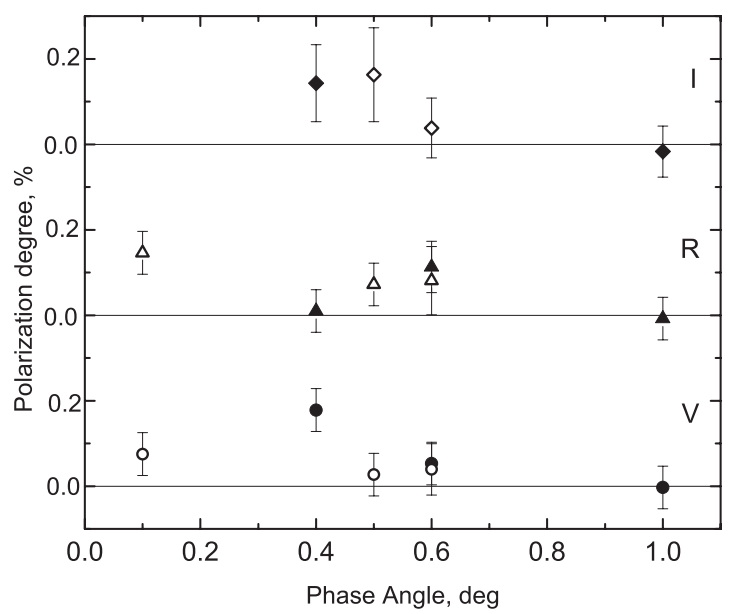

Fig. 7. Polarization of 1021 Flammario at small phase angles. Open and filled symbols represent, respectively, observations before and after the asteroid's opposition.

the phase angle close to the angle of minimum polarization (Fig. 6). Such a polarization degree implies moderate surface albedo for this asteroid, in contradiction with its IRAS albedo of 0.042 . An error in the albedo determination is unlikely since Bertholda belongs to the Cybele group populated by low albedo asteroids. The probable explanation is that we observed a decrease in the depth of the negative polarization branch for very dark surfaces, previously found for the asteroid 419 Aurelia, which has an IRAS albedo of 0.046 (Belskaya et al. 2005).

We also made the first polarimetric observations of a D-type asteroid, showing that the polarization value of the unusual minor planet 944 Hidalgo is similar to that of low albedo asteroids (Fig. 6).

\subsection{Small phase angle observations of 1021 Flammario}

Detailed VRI observations of the low albedo asteroid Flammario have been carried out at extremely small phase angles down to $0.1^{\circ}$ (Table 2). These are the first measurements in such detail near opposition for a low albedo asteroid. Measured values are shown in Fig. 7, where observations before and after opposition are marked by different symbols. The observations show zero or slightly positive polarization at small phase angles in all filters, both before and after opposition. The observations at the same phase angle of $0.6^{\circ}$ made before and after opposition are in very good agreement. The values of $Q$ and $U$ are close to each other at the same phase angles, indicating a $45^{\circ}$ deviation of the polarization plane from the scattering plane. This unusual characteristic could be attributed to an instrumental effect, or else it could be related to physical properties of the asteroid surface. Although the polarization degree is very small in the phase angle range, given the high accuracy of our calibration we exclude a systematic error. We favor an explanation related to the effect of surface anisotropy, e.g., an anisotropy caused by grooves, such us the Phobos grooves. Laboratory measurements (Geake et al. 1984) have shown that this explanation is physically sound.

\section{Conclusions}

Using the AFOSC polarimeter, we have greatly expanded the number of objects observed with high accuracy. Our survey includes near-Earth and main belt asteroids, as well as a Jupitercrossing object, for a total of 36 objects. The main portion of the 
obtained polarimetric values is within the typical range of average polarization-phase curves of a corresponding taxonomic class. Our results prove that most asteroids have a rather uniform surface microstructure. Only five asteroids in our data set, namely 71 Niobe, 152 Atala, 420 Bertholda, 785 Zwetana, and 1627 Ivar, are outside the typical values of their classes. This result can derive either from an erroneous taxonomic classification or from their particular surface properties. Further data are needed to clarify the situation.

Small phase angle observations of the asteroid 1021 Flammario show abnormal positive polarization in the range of phase angles $0.1-1.0^{\circ}$, which can be attributed to surface structure anisotropy of the asteroid.

Acknowledgements. We thank Dr. A. Della Valle for his help in performing part of the observations

\section{References}

Barucci, M. A., Fulchignoni, M., Fornasier, S., et al. 2005, A\&A, 430, 313

Belskaya, I. N., \& Lagerkvist, C. I. 1996, Planet. Space Sci., 44, 783

Belskaya, I. N., Efimov, Ju. S., Lupishko, D. F., \& Shakhovskoj, N. M. 1985, Sov. Astron. Lett., 11, 116

Belskaya, I. N., Kiselev, N. N., Lupishko, D. F., \& Chernova, G. P. 1987a, Kinemat. Phys. Neb. Tel, 3, 19

Belskaya, I. N., Lupishko, D. F., \& Shakhovskoj, N. M. 1987b, Sov. Astron. Lett., 13, 219

Belskaya, I. N., Kiselev, N. N., Lupishko, D. F., \& Chernova, G. P. 1991, Kinemat. Phys. Neb. Tel, 7, 11

Belskaya, I. N., Shevchenko, V. G ., Kiselev, N. N., et al. 2003, Icarus, 166, 276 Belskaya, I. N., Shkuratov, Yu. G., Efimov, Yu. S., et al. 2005, Icarus, 178, 213

Birlan, M., Barucci, M. A., Vernazza, P., et al. 2004, New A, 9, 343

Broglia, P., \& Manara, A. 1990, A\&A, 237, 256

Broglia, P., \& Manara, A. 1992, A\&A, 257, 770

Broglia, P., Manara, A., \& Farinella, P. 1994, Icarus, 109, 204

Bus, B., \& Binzel, R. P. 2002, Icarus, 158, 146
Cellino, A., Hutton, R. G., Tedesco, E. F., di Martino, M., \& Brunini, A. 1999, Icarus, 138, 129

Cellino, A., Hutton, R. G., di Martino, M., et al. 2005, Icarus, 179, 304

Chernova, G. P., Lupishko, D. F., \& Shevchenko, V. G. 1994, Kinemat. Phys. Neb. Tel, 10, 45

Delbó, M., Harris, A. W., Binzel, R. P., \& Pravec, P. 2003, Icarus, 166, 116

Desidera, S., Giro, E., Munari, U., et al. 2004, A\&A, 414, 591

Fornasier, S., Belskaya, I., Fulchignoni, M., Barucci, M. A., \& Barbieri, C. 2006, A\&A, 449, 9

Geake, J., Geake, M., \& Zellner, B. 1984, MNRAS, 210, 89

Gehrels, T., 1974, in Planets, stars and nebulae studied with photopolarimetry, ed. T. Gehrels (Tucson: University of Arizona Press), 3

Kiselev, N. N., Chernova, G. P., \& Lupishko, D. F. 1994, Kinematika. Fiz. Nebesnyka Tel., 10, 35

Licandro, J. 2005, personal communication

Lupishko, D. F. \& Mohamed, R. A. 1996, Icarus, 119, 209

Lupishko, D. F., Kiselev, N. N., Chernova, G. P., Shakhovskoj, N. M., \& Vasilyev, S. V. 1994, Kinemat. Phys. Neb. Tel, 10, 40

Magri, C., Ostro, S. J., Rosema, D. K., et al. 1999, Icarus, 140, 379

Muinonen, K. 2004, Waves in Random Media, 14, 365

Muinonen, K., Piironen, J., Shkuratov, Yu. G., Ovcharenko, A., \& Clark, B. E. 2002, in Asteroids III, ed. W. Bottke, R. P. Binzel, A. Cellino, \& P. Paolicchi (Tucson: University of Arizona Press), 1

Pentilla, A., Lumme, K., Hadamcik, E., \& Levasseur-Regourd, A. C. 2005, A\&A, 432, 1081

Pernechele, C., Fantinel, D., \& Giro, E. 2003, SPIE Proc., 4843, 156

Rivkin, A. S., Howell, E. S., Lebofsky, L. A., et al. 2000, Icarus, 145, 351

Shakhovskoy, N. M., \& Efimov, Yu. S. 1972, Izv. Krymskoi Astrofiz. Obs., 45, 90

Serkowski, K. 1974, in Planets, stars and nebulae studied with photopolarimetry, ed. T. Gehrels (Tucson: University of Arizona Press), 135

Shkuratov, Yu. G., Ovcharenko, A., Zubko, E., et al. 2002, Icarus, 159, 396 Shkuratov, Yu., Ovcharenko, A., Zubko, E., et al. 2004, J. Quant. Spectr. Rad. Trans., 88, 267

Tedesco, E. F., Noah, P. V., Noah, M., \& Price, S. D. 2002, AJ, 123, 1056

Tholen, D. J. 1985, IAU Circ., 4034

Tholen, D. J. 1989, in Asteroids III, ed. W. Bottke, R. P. Binzel, A. Cellino, \& P. Paolicchi (Tucson: University of Arizona Press), 1139

Volten, H., Munoz, O., Rol, E., et al. 2001, J. Geophys. Res., 106, Iss. 17, 375

Zellner, B., \& Gradie J. 1976, AJ, 81, 262

Zubko, E., Petrov, D., Shkuratov, Y., \& Videen, G. 2005, Appl. Optics, 44, 6479 